\title{
Dual-wavelength photo-killing of methicillin-resistant Staphylococcus aureus
}

\author{
Leon G. Leanse, ${ }^{1,2}$ Xueping Sharon Goh, ${ }^{1,2}$ Ji-Xin Cheng, ${ }^{4}$ David C. Hooper, ${ }^{3}$ and Tianhong Dai ${ }^{1,2}$ \\ 'Wellman Center for Photomedicine, ${ }^{2}$ Vaccine and Immunotherapy Center, and ${ }^{3}$ Division of Infectious Diseases, \\ Massachusetts General Hospital, Harvard Medical School, Boston, Massachusetts, USA. ${ }^{4}$ Department of Chemistry, \\ Boston University, Boston, Massachusetts, USA.
}

\begin{abstract}
With the effectiveness of antimicrobials declining as antimicrobial resistance continues to threaten public health, we must look to alternative strategies for the treatment of infections. In this study, we investigated an innovative, drug-free, dual-wavelength irradiation approach that combines 2 wavelengths of light, $460 \mathrm{~nm}$ and $\mathbf{4 0 5} \mathrm{nm}$, against methicillin-resistant Staphylococcus aureus (MRSA). MRSA was initially irradiated with 460-nm light $\left(90-360 \mathrm{~J} / \mathrm{cm}^{2}\right)$ and subsequently irradiated with aliquots of $405-\mathrm{nm}$ light $\left(54-324 \mathrm{~J} / \mathrm{cm}^{2}\right)$. For in vivo studies, mouse skin was abraded and infected with approximately $10^{7}$ CFUs of MRSA and incubated for 3 hours before irradiating with $460 \mathrm{~nm}\left(360 \mathrm{~J} / \mathrm{cm}^{2}\right)$ and $405 \mathrm{~nm}\left(342 \mathrm{~J} / \mathrm{cm}^{2}\right)$. Naive mouse skin was also irradiated to investigate apoptosis. We found that staphyloxanthin, the carotenoid pigment in MRSA cells, promoted resistance to the antimicrobial effects of 405-nm light. In addition, we found that the photolytic effect of 460-nm light on staphyloxanthin attenuated resistance of MRSA to 405-nm light killing. Irradiation of $\mathbf{4 6 0} \mathbf{n m}$ alone did not elicit any antimicrobial effect on MRSA. In a proofof-principle mouse skin abrasion infection model, we observed significant killing of MRSA using the dual-wavelength irradiation approach. However, when either wavelength of light was administered alone, no significant decrease in bacterial viability was observed. Moreover, exposure of the dualwavelength irradiation to naive mouse skin did not result in any visible apoptosis. In conclusion, a dual-wavelength irradiation strategy may offer an innovative, effective, and safe approach for the treatment of skin infections caused by MRSA.
\end{abstract}

Conflict of interest: The authors have declared that no conflict of interest exists.

Copyright: () 2020, American Society for Clinical Investigation.

Submitted: October 17, 2019

Accepted: April 23, 2020

Published: June 4, 2020.

Reference information: /CI Insight. 2020;5(11):e134343.

https://doi.org/10.1172/jci.

insight.134343.

\section{Introduction}

In recent years, the threat of antimicrobial resistance has become one of the most important concerns for public health. Infection outbreaks that result from multidrug-resistant organisms that have emerged remain a significant problem $(1,2)$. Staphylococcus aureus infections are among the most important causes of skin and soft tissue infections, with methicillin-resistant Staphylococcus aureus (MRSA) being particularly important $(3,4)$. Therefore, novel and nontraditional approaches must be explored to quell these negative effects. Over the years, antimicrobial blue light (aBL) at a 405-nm wavelength has been emerging as a potential alternative treatment for localized infections (5). The accepted mechanism responsible for the antimicrobial effects of aBL $(405 \mathrm{~nm})$ is through excitation of endogenous photosensitizing porphyrins and the subsequent generation of singlet oxygen, resulting in lipid peroxidation, DNA damage, cell wall damage, and cellular apoptosis of microbial cells (5). However, previous studies and preliminary results in our laboratory showed that MRSA is much more tolerant of aBL at $405 \mathrm{~nm}$ than most other species (6). Recent findings by other groups have demonstrated the antioxidant properties of staphyloxanthin (STX), which is a membrane-bound carotenoid pigment of MRSA, responsible for its characteristic golden colony phenotype $(7,8)$. In addition, it has been demonstrated that STX is subject to photolysis through 460-nm light exposure, rendering it more susceptible to $\mathrm{H}_{2} \mathrm{O}_{2}$-mediated killing (9). Therefore, we theorized that the limited antimicrobial efficacy of aBL (405 nm) we observed with MRSA was a direct result of STX because this is also a known singlet oxygen scavenger. As a result, we hypothesized that treatment of MRSA using 405-nm light could be improved by STX photolysis using preexposure with 460-nm light. Here we report a potentially novel dual-wavelength irradiation approach using the combination of 460-nm and 405-nm light, which exploited the STX photolytic effect of 460-nm light to sensitize MRSA to 405-nm light. 


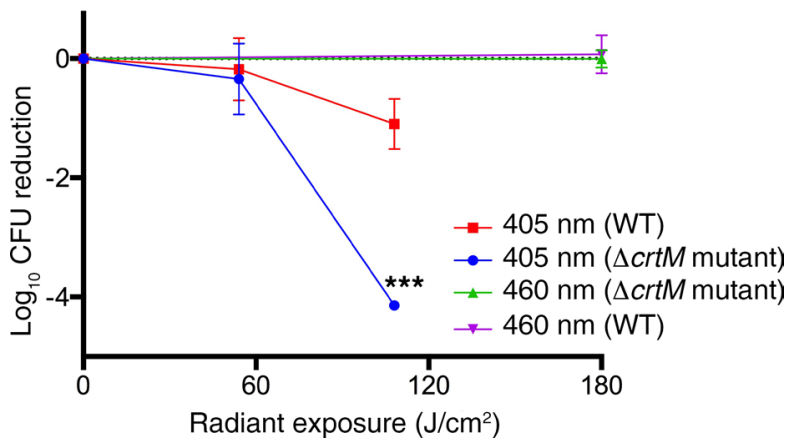

Figure 1. STX promoted resistance in S. aureus to 405-nm light-mediated killing. Graphs illustrate the killing kinetics of S. aureus Pig1 WT and the $\Delta c r t M$ mutant by $54 \mathrm{~J} / \mathrm{cm}^{2}$ and $108 \mathrm{~J} / \mathrm{cm}^{2} 405-\mathrm{nm}$ light or $460-\mathrm{nm}$ light $\left(180 \mathrm{~J} / \mathrm{cm}^{2}\right)$. Error bars represent SEM $(n=3)$. ${ }^{* *} P \leq 0.001$; paired $t$ test comparing $405 \mathrm{~nm}(\mathrm{WT})$ versus $405 \mathrm{~nm}(\Delta \mathrm{crtM})$ mutant.

\section{Results}

STX promoted resistance in S. aureus to 405-nm light-mediated killing. In this study, we investigated whether the presence of STX within MRSA is responsible for its inherent resistance to aBL (405 nm). We compared the killing potential of a WT $S$. aureus strain (Pig1) that produces STX and an $\triangle$ crtM isogenic mutant (Pig1 $\triangle c r t M)$ of the same strain that is deficient in the production of STX. After $108 \mathrm{~J} / \mathrm{cm}^{2} \mathrm{aBL}, \mathrm{a} 4.14 \log _{10} \mathrm{CFU}$ reduction was achieved in Pig1 $\triangle c r t M$, compared with its WT parental strain in which only a $1.09 \log _{10}$ CFU reduction was observed $(P<0.001$; Figure 1). These findings thus demonstrated the ability for STX to promote resistance to aBL at $405 \mathrm{~nm}$.

Exposure to 460-nm light sensitized MRSA to 405- $\mathrm{nm}$ light. A recent study discovered that $460 \mathrm{~nm}$ irradiation can lyse the STX pigment, rendering MRSA more sensitive to ROS-mediated killing (9). Therefore, we sought to determine whether pre-irradiation with $460-\mathrm{nm}$ light was able to attenuate STX-mediated resistance of MRSA to 405-nm light killing. In the strain MRSA USA300, we found that $108 \mathrm{~J} / \mathrm{cm}^{2}$ exposure of $405-\mathrm{nm}$ light was only capable of inactivating $0.64 \log _{10}$ CFUs (Figure 2A). However, preexposure to $460-\mathrm{nm}$ light $\left(180 \mathrm{~J} / \mathrm{cm}^{2}\right)$ significantly potentiated the killing of MRSA with a $3.58 \log _{10} \mathrm{CFU}$ reduction $(P=0.004 ;$ Figure 2A). Irradiation with $460 \mathrm{~nm}$ alone, at an equivalent dose, was insufficient to elicit any antimicrobial effects. In a clinical strain of MRSA (AF0003), we also observed a significant improvement in the killing efficacy of 405-nm light with a preexposure of 460-nm light. aBL (405 nm) alone inactivated $1.83 \log _{10}$ CFUs, whereas the addition of $460-\mathrm{nm}$ light resulted in a $3.23 \log _{10}$ CFU reduction $(P=0.02$; Figure 2B). As with MRSA USA300, 460-nm irradiation alone, at an equivalent dose, was insufficient to elicit any antimicrobial effects on the strain AF0003.

STX photolysis alone mediated sensitization of MRSA to 405- $n$ m light. To determine whether the enhancement in MRSA CFU reduction by 460-nm light was solely based on STX photolysis or whether there was another underlying mechanism, Pig1 $\triangle c r t M$ and its parental WT strain were exposed to 405-nm light or the $460 \mathrm{~nm}+405 \mathrm{~nm}$ combination. No significant difference in CFU reduction was identified when the $S$. aureus Pig1 $\Delta c r t M$ mutant was exposed to dual-wavelength irradiation (460-nm light at 180 $\mathrm{J} / \mathrm{cm}^{2}$ and $405 \mathrm{~nm}$ at $54 \mathrm{~J} / \mathrm{cm}^{2}$ or $108 \mathrm{~J} / \mathrm{cm}^{2}$ ), compared with 405-nm light exposure $\left(54 \mathrm{~J} / \mathrm{cm}^{2}\right.$ or 108 $\mathrm{J} / \mathrm{cm}^{2}$ ) alone (Figure 3A; $P=0.24$ ), suggesting that the role of $460-\mathrm{nm}$ light is solely to photolyze STX and subsequently render MRSA more susceptible to ROS generated by $405-\mathrm{nm}$ light. With respect to the WT strain, preexposure to $460-\mathrm{nm}$ light $\left(180 \mathrm{~J} / \mathrm{cm}^{2}\right)$ significantly enhanced $405-\mathrm{nm}$ aBL killing, further confirming the role of 460-nm light in sensitizing MRSA to 405-nm aBL ( $P=0.001$; Figure $3 \mathrm{~B})$.

Enhancement of 405-nm light killing by 460-nm light was dose dependent. In this study, we determined the effect of increasing radiant exposures of 460-nm light on the effectiveness of 405-nm light. The representative clinical strain of MRSA (AF0003) was used. MRSA was exposed to different radiant exposures of $460-\mathrm{nm}$ light $\left(90 \mathrm{~J} / \mathrm{cm}^{2}, 180 \mathrm{~J} / \mathrm{cm}^{2}\right.$, or $360 \mathrm{~J} / \mathrm{cm}^{2}$; reflecting a preexposure duration of 15,30 , and 60 minutes, respectively), before exposing bacteria to $54 \mathrm{~J} / \mathrm{cm}^{2}$ or $108 \mathrm{~J} / \mathrm{cm}^{2}$ of $405-\mathrm{nm}$ light. Preexposure to $360 \mathrm{~J} / \mathrm{cm}^{2}$ of $460-\mathrm{nm}$ light resulted in the most significant killing of MRSA after $54 \mathrm{~J} / \mathrm{cm}^{2}$ of $405-\mathrm{nm}$ light, with a killing of $2.14 \log _{10}$ CFUs compared with $405 \mathrm{~nm}$ alone, which only reduced the CFUs by 0.36 $\log _{10}$ CFUs ( $P=0.002$; Figure 4). Conversely, exposing MRSA to $54 \mathrm{~J} / \mathrm{cm}^{2}$ of 405 -nm light after exposure to $90 \mathrm{~J} / \mathrm{cm}^{2}$ or $180 \mathrm{~J} / \mathrm{cm}^{2}$ did not result in any significant improvement $(P=0.9)$. This suggests that the 

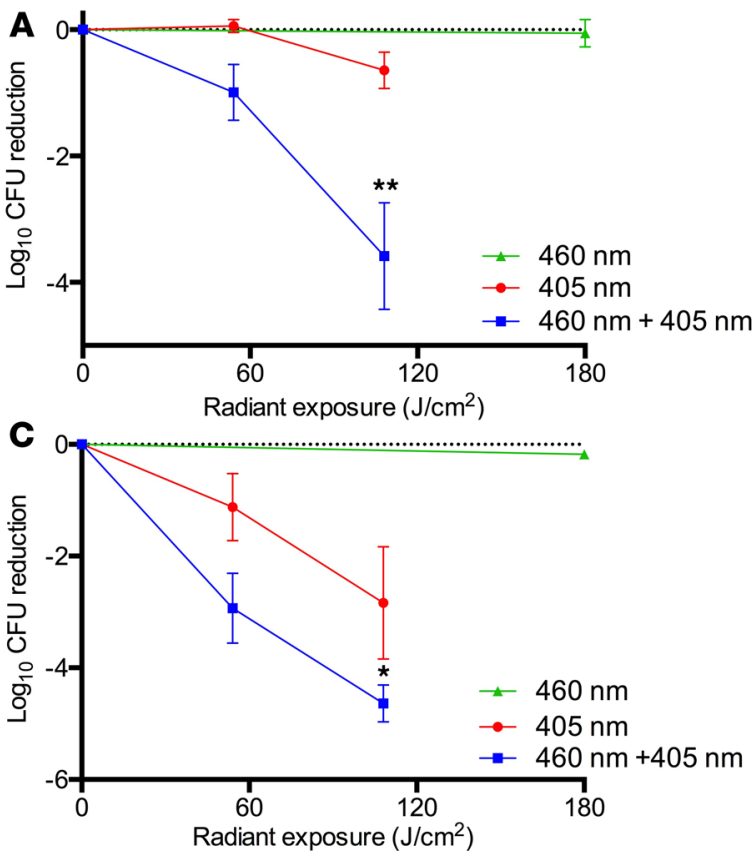

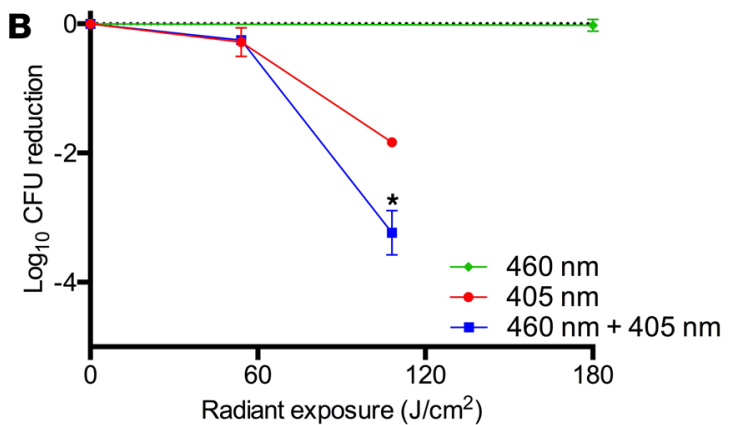

Figure 2. Exposure to 460-nm light sensitized MRSA to 405-nm light. Graphs illustrate the killing kinetics of (A) MRSA USA300, (B) MRSA AF0003, and (C) MRSA IQ00064 by $405-\mathrm{nm}$ light $\left(54 \mathrm{~J} / \mathrm{cm}^{2}\right.$ or $\left.108 \mathrm{~J} / \mathrm{cm}^{2}\right)$, $460 \mathrm{~nm}+460-\mathrm{nm}$ light (preexposure to $180 \mathrm{~J} / \mathrm{cm}^{2} 460-\mathrm{nm}$ light, followed by $54 \mathrm{~J} / \mathrm{cm}^{2}$ or $108 \mathrm{~J} / \mathrm{cm}^{2} 405-\mathrm{nm}$ light), or $460-\mathrm{nm}$ light $\left(180 \mathrm{~J} / \mathrm{cm}^{2}\right)$. Error bars represent SEM $(n=3)$. ${ }^{*} P \leq 0.05,{ }^{*} P \leq 0.01$ (paired $t$ test comparing $405 \mathrm{~nm}$ vs. $460 \mathrm{~nm}+460 \mathrm{~nm})$

enhancement of the antimicrobial efficacy of $405-\mathrm{nm}$ light by $460-\mathrm{nm}$ light is dose dependent, which is not surprising because increased photolysis of STX by $360 \mathrm{~J} / \mathrm{cm}^{2}$ may have increased the sensitivity of MRSA to lower 405-nm doses. When exposure to $405-\mathrm{nm}$ aBL was increased to $108 \mathrm{~J} / \mathrm{cm}^{2}$, preexposure to $180 \mathrm{~J} /$ $\mathrm{cm}^{2}$ significantly enhanced killing of MRSA relative to $405-\mathrm{nm}$ light alone $(P=0.02)$. At a radiant exposure of $90 \mathrm{~J} / \mathrm{cm}^{2}$ of $460-\mathrm{nm}$ light, however, there was no significant improvement in MRSA killing when the exposure was $108 \mathrm{~J} / \mathrm{cm}^{2}$ of $405-\mathrm{nm}$ light $(P=0.40)$. These findings suggest that enhancement of $405-\mathrm{nm}$ light-mediated killing by preexposure to $460-\mathrm{nm}$ light was contingent on the delivered radiant exposure of 460-nm light, in that increasing radiant exposures of 460-nm light resulted in increased susceptibility of MRSA to 405-nm light. We additionally found there to be some increase in intracellular ROS (1.56-fold) when $405-\mathrm{nm}$ light was administered after photolysis by $460-\mathrm{nm}$ light $\left(360 \mathrm{~J} / \mathrm{cm}^{2}\right)$ when compared with $405 \mathrm{~nm}$ alone; however, this was not found to be statistically significant ( $P=0.24$; Supplemental Figure 2; supplemental material available online with this article; https://doi.org/10.1172/jci.insight.134343DS1).

Dual-wavelength 460-nm $+405-\mathrm{nm}$ light exposure effectively reduced the viability of MRSA biofilms. In this study, we investigated whether preexposure to 460-nm light improved the efficacy of $405-\mathrm{nm}$ aBL against 48-hour-old MRSA biofilms. The MRSA AF0003 strain was used as the representative strain for all biofilm experiments. The biofilms were initially exposed to $180 \mathrm{~J} / \mathrm{cm}^{2}$ of $460-\mathrm{nm}$ light immediately before exposing them to $405-\mathrm{nm}$ light at $108 \mathrm{~J} / \mathrm{cm}^{2}, 216 \mathrm{~J} / \mathrm{cm}^{2}$, or $324 \mathrm{~J} / \mathrm{cm}^{2}$. We found that irradiation of $108 \mathrm{~J} / \mathrm{cm}^{2}$ of aBL at $405 \mathrm{~nm}$ did not result in any antimicrobial effects in the dual-wave-
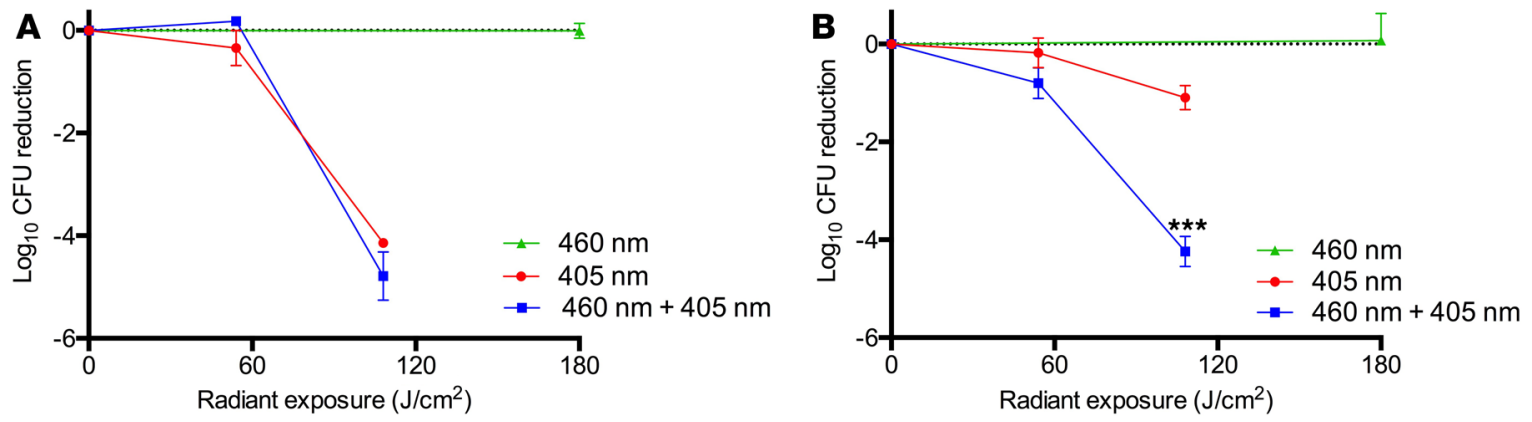

Figure 3. STX photolysis alone mediated sensitization of MRSA to 405-nm light. Graphs illustrate the killing kinetics of (A) S. aureus Pig1 $\triangle \mathrm{crtM}$ mutant and (B) S. aureus Pig1 (WT strain) with $405 \mathrm{~nm}, 460 \mathrm{~nm}+460 \mathrm{~nm}$, or $460 \mathrm{~nm}$. Error bars represent SEM $(n=3)$. ***P $P 0.001$ (paired $t$ test comparing 405 nm vs. $460 \mathrm{~nm}+460 \mathrm{~nm})$. 


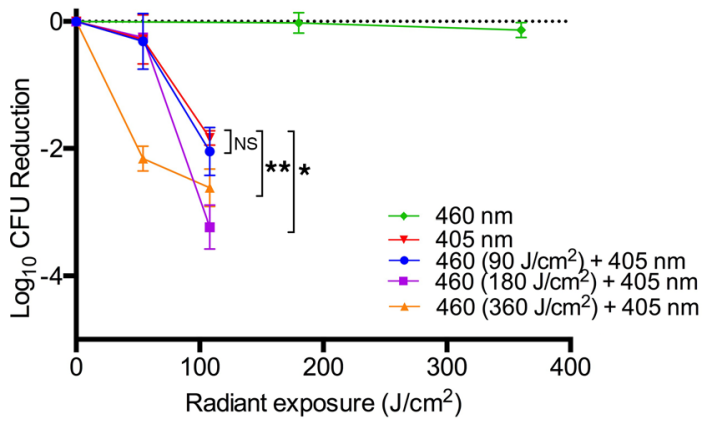

Figure 4. Enhancement of 405-nm light killing by 460-nm light was dose dependent. Graph illustrates the killing kinetics of MRSA by $405-\mathrm{nm}$ light after radiant exposures of $54 \mathrm{~J} / \mathrm{cm}^{2}$ or $108 \mathrm{~J} / \mathrm{cm}^{2}$, with or without preexposure to $90 \mathrm{~J} / \mathrm{cm}^{2}, 180 \mathrm{~J} / \mathrm{cm}^{2}$, or $360 \mathrm{~J} / \mathrm{cm}^{2}$. Error bars represent SEM $(n=3) .{ }^{*} P \leq 0.05,{ }^{* *} P \leq 0.01$ (paired $t$ test comparing $405 \mathrm{~nm}$ vs. $460 \mathrm{~nm}+460 \mathrm{~nm}$ ).

length irradiation-exposed group, or in the 405-nm light alone-treated group (Figure 5). When the exposure of $405-\mathrm{nm}$ light was increased to $216 \mathrm{~J} / \mathrm{cm}^{2}$, however, the dual-wavelength irradiation-treated group showed a CFU reduction of $1.85 \log _{10}$, compared with a $0.73 \log _{10}$ CFU reduction in the $405-$ $\mathrm{nm}$ light alone-treated group $(P<0.05)$. When the exposure of $405-\mathrm{nm}$ light reached $324 \mathrm{~J} / \mathrm{cm}^{2}$, the dual-wavelength irradiation resulted in a $2.72 \log _{10}$ CFU reduction in MRSA biofilms compared with 405-nm light alone, which inactivated $1.49 \log _{10}$ CFUs $(P=0.002$; Figure 5).

Dual-wavelength of 460-nm + 405-nm light significantly improved the treatment efficacy in a mouse skin abrasion infection model. To predict the clinical translatability of the dual-wavelength irradiation approach combining 460-nm and 405-nm light exposure, we investigated its efficacy in a proof-of-principle mouse skin abrasion infection model. Mice were inoculated within an abrasion wound with approximately $10^{7} \mathrm{CFUs}$ of MRSA USA300 and incubated 3 hours before treatment with the dual-wavelength irradiation. When the infected wounds were treated with the dual-wavelength irradiation $\left(460 \mathrm{~nm} / 342 \mathrm{~J} / \mathrm{cm}^{2}\right.$ and $405 \mathrm{~nm} / 360 \mathrm{~J} / \mathrm{cm}^{2}$ ), a $1.97 \log _{10}$ CFU reduction was achieved, compared with $405 \mathrm{~nm}$ alone at an equivalent radiant exposure, which only reduced the CFUs by $0.28 \log _{10}$ CFUs $(P<0.0001$; Figure 6; $n=7)$. Treatment with $460-\mathrm{nm}$ light $\left(342 \mathrm{~J} / \mathrm{cm}^{2}\right)$ alone did not influence bacterial viability within the wounds. These findings demonstrated the potential of the dual-wavelength $(460 \mathrm{~nm}+405 \mathrm{~nm})$ irradiation approach as a viable treatment option for wound infections caused by MRSA.

Dual-wavelength exposure of 460-nm $+405-\mathrm{nm}$ light did not result in apoptosis of mouse skin cells. To determine whether the dual-wavelength irradiation by the combination 460-nm and 405-nm light results in cellular apoptosis of mammalian skin cells, the therapeutic dose of the dual-wavelength irradiation used during the in vivo killing studies (460-nm light/342 J/ $\mathrm{cm}^{2}$ and 405-nm light/t $360 \mathrm{~J} /$ $\mathrm{cm}^{2}$ ) was administered to naive mouse skin tissue before assessing cellular apoptosis using the TUNEL assay. The results showed no presence of apoptotic cells in the treated group immediately after the dual-wavelength irradiation treatment, 24 hours after treatment, or 48 hours after treatment (Figure 7, A-C). The untreated group did not show any evidence of apoptosis (Figure 7D) compared with the positive control (Figure 7E).

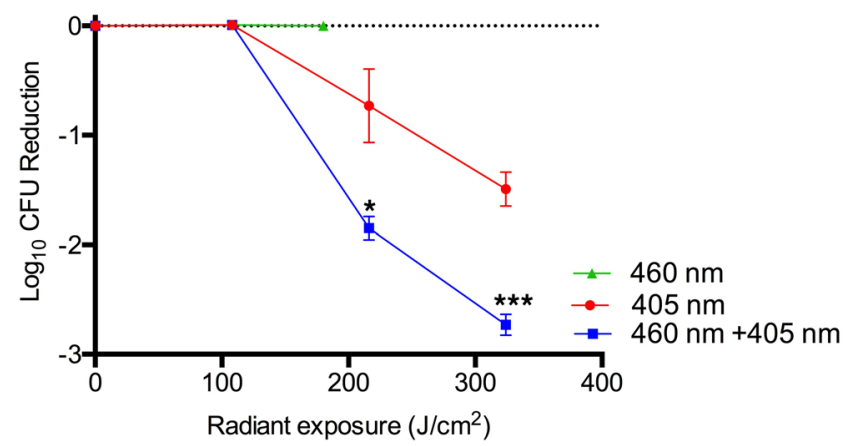

Figure 5. Dual-wavelength 460-nm + 405-nm light exposure effectively reduced the viability of MRSA biofilms. Graph illustrates the killing kinetics of MRSA AF0003 48-hour biofilms after exposure to 405-nm light (108 J/cm², $216 \mathrm{~J} / \mathrm{cm}^{2}$, or $324 \mathrm{~J} / \mathrm{cm}^{2}$ ), 460-nm + 460-nm light (preexposure to $180 \mathrm{~J} / \mathrm{cm}^{2} 460-\mathrm{nm}$ light, followed by $108 \mathrm{~J} / \mathrm{cm}^{2}, 216 \mathrm{~J} /$ $\mathrm{cm}^{2}$, and $\left.324 \mathrm{~J} / \mathrm{cm}^{2}\right)$, or $460-\mathrm{nm}$ light $\left(180 \mathrm{~J} / \mathrm{cm}^{2}\right)$. Error bars represent SEM $(n=3)$. ${ }^{*} P \leq 0.05,{ }^{* * *} P \leq 0.001$ (paired $t$ test comparing $405 \mathrm{~nm}$ vs. $460 \mathrm{~nm}+460 \mathrm{~nm})$. 


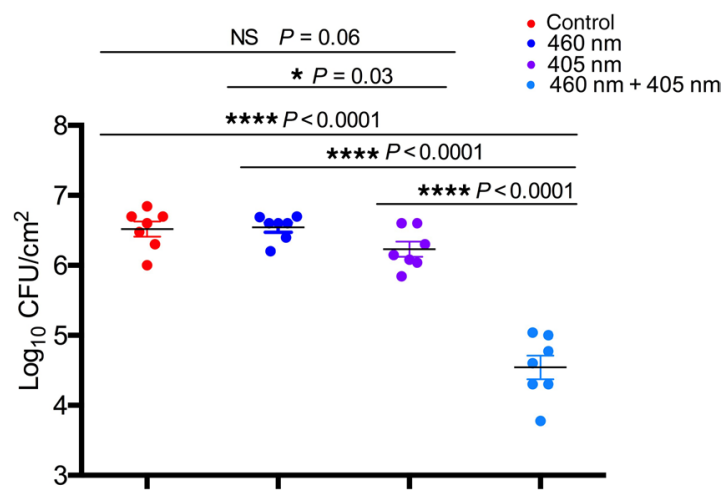

Figure 6. Scatter plot showing $\log _{10} \mathrm{CFU} / \mathrm{cm}^{2}$ after no treatment (control), 460-nm light treatment, 405-nm light treatment, and $\mathbf{4 6 0} \mathbf{n m}+\mathbf{4 0 5}-\mathbf{n m}$ light treatment. Each point represents an individual value; the mean value is represented by the horizontal line. Error bars represent SEM $(n=7)$. The statistical methods employed were a paired $t$ test comparing 2 individual conditions ( $405 \mathrm{~nm}$ vs. $460 \mathrm{~nm}+405 \mathrm{~nm} ; 405 \mathrm{~nm}$ vs. $460 \mathrm{~nm}$ ), and a 1-way ANOVA comparing 3 or more groups (highlighted by the horizontal lines spanning the different groups).

\section{Discussion}

In this study, we investigated the role of STX in promoting resistance to 405-nm light killing in MRSA. In addition, we explored the STX photolytic effect of 460-nm light exposure as a means of sensitizing MRSA to 405-nm light killing. For the first time to our knowledge, with the use of STX-deficient Pig1 $\Delta c r t M$, we confirmed that the presence of STX within MRSA was directly responsible for the relative resistance phenotype to 405-nm light that has been observed in our and other laboratories (6). There have, however, been studies that have illustrated the sensitivity of 405-nm light killing, which conflict somewhat with ours (10). It is possible that variabilities in the relative abundance of endogenous STX may explain these findings. Furthermore, because STX is present in $90 \%$ of strains (11), it is feasible that improved efficacy of $405-\mathrm{nm}$ light may be achieved using these STX-deficient strains, although further work is required to corroborate this hypothesis. Given that STX has a carotenoid structure, and is thus a singlet oxygen scavenger, it is unsurprising that its presence would interfere with damage elicited by singlet oxygen (12). Liu et al. fully characterized the role of STX and found it to be a powerful singlet oxygen scavenger (7). They found that the STX-deficient isogenic mutant $\Delta c r t M$ was greater than 100 -fold more susceptible to singlet oxygen generated through methylene blue-mediated photodynamic therapy (MB-PDT), relative to its parental WT (7). These findings, therefore, strongly support our findings illustrating the role of STX in promoting resistance to $405 \mathrm{~nm}$-mediated killing. In a previous study, it was demonstrated that photolysis using 460-nm light sensitized MRSA to killing by hydrogen peroxide (9), further supporting the role of STX in eliciting resistance to ROS resulting from 405-nm light illumination.

We next sought to explore whether preexposure to $460-\mathrm{nm}$ light would be adequate to overcome the relative resistance observed when MRSA is exposed to 405-nm light. It was demonstrated previously that 460-nm light illumination was capable of STX lysis in MRSA. We found that the degree of STX photolysis achieved by 460-nm light illumination significantly potentiated the killing of MRSA when subsequently exposed to $405 \mathrm{~nm}$, suggesting that the extent of STX lysis was enough to overcome any innate resistance to 405 -nm light killing.

Exposure to 460-nm light alone, however, was insufficient to elicit any antimicrobial effects. Our results are consistent with findings from other studies that found 460-nm light did not significantly influence the viability of MRSA in vitro $(6,9)$. In contrast, however, findings from another study demonstrated that at a wavelength of $470 \mathrm{~nm}$, a significant loss of viability may be achieved in $S$. aureus (13). Furthermore, a recent study found that combining riboflavin with 450-nm light potentiated the killing of MRSA (14). Riboflavin has been shown to possess photosensitizing properties when activated with UV to eliminate microorganisms $(15,16)$. In addition, riboflavin has been found to be effective in combination with $460-\mathrm{nm}$ light for inactivating Listeria monocytogenes (17), which is not surprising given that riboflavin can absorb light at this wavelength (18). Moreover, MRSA can produce riboflavin endogenously depending on the exogenous availability of riboflavin (19). A study by Chaffin et al. found that genes encoding riboflavin biogenesis were not upregulated when riboflavin was adequately available (such as when grown in Luria-Bertani medium; ref. 20); suggesting that 
A

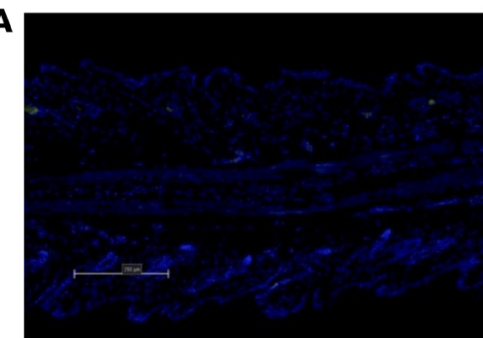

B

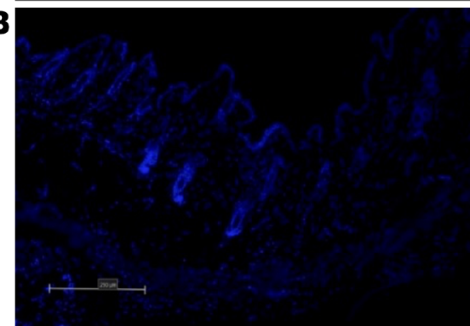

C

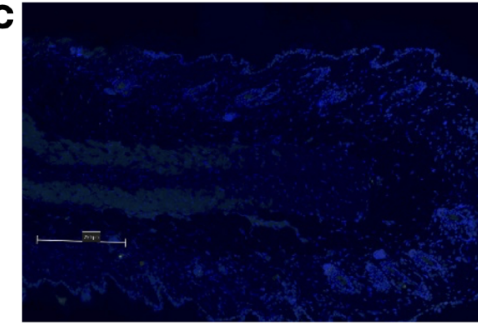

D

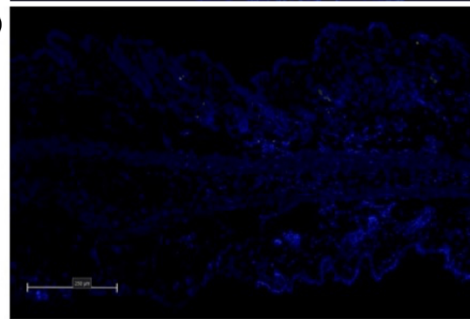

E

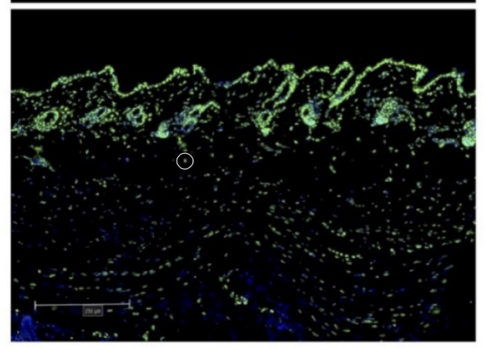

Figure 7. Dual-wavelength exposure of 460-nm + 405-nm light did not result in apoptosis of mouse skin cells. Representative images showing TUNEL-stained mouse skin sections for the detection of apoptotic cells resulting from the following treatments. (A) Zero hours after treatment with 460-nm light $\left(342 \mathrm{~J} / \mathrm{cm}^{2}\right)+405-\mathrm{nm}$ light $\left(360 \mathrm{~J} / \mathrm{cm}^{2}\right)$, (B) 24 hours after treatment with 460-nm light $\left(342 \mathrm{~J} / \mathrm{cm}^{2}\right)+405-\mathrm{nm}$ light $\left(360 \mathrm{~J} / \mathrm{cm}^{2}\right)$, (C) 48 hours after treatment with 460-nm light (342 $\left.\mathrm{J} / \mathrm{cm}^{2}\right)+405-\mathrm{nm}$ light $\left(360 \mathrm{~J} / \mathrm{cm}^{2}\right)$, and (D) no treatment. (E) A positive control treated with DNase I was also added. Florescence of fluorescein and DAPI are represented by green (indicative of fluorescein binding to damaged DNA) and blue (DAPI stain of intact nuclei) pseudo-color, respectively. DAPI is a nuclear counterstain. White circle indicates apoptotic cell indicated by green fluorescence. Scale bar: $250 \mu \mathrm{m}$.

when exogenous riboflavin is limited, MRSA may overproduce riboflavin endogenously. Therefore, it is reasonable to infer that variabilities in the endogenous production of riboflavin may offer an explanation of the conflicting findings achieved by different studies into the antimicrobial effects of $450-\mathrm{nm}$ to $470-\mathrm{nm}$ light.

In addition to antimicrobial killing with the use of light within the blue light spectrum (i.e., $400 \mathrm{~nm}-470 \mathrm{~nm}$ ), there have been other light-based modalities that have effectively eliminated organisms. For example, MB-PDT, a commonly used light-based antimicrobial approach (which uses a photosensitizer $[\mathrm{MB}]$ that is activated by red light to generate ROS), has been used against an array of bacterial pathogens, inclusive of MRSA in vitro and in vivo $(21,22)$. A study by Vecchio et al. found that MB-PDT was able reduce the relative luminescence of a bioluminescent variant of MRSA USA300 inoculated onto a burn (30-minute incubation) by approximately 2 $\log _{10}$ CFUs (22). This reduction in MRSA was similar to what we observed after 3 hours of incubation with MRSA USA300 using the 460-nm $+405-\mathrm{nm}$ light approach. The obvious benefit of our $460-\mathrm{nm}+405-\mathrm{nm}$ light approach is that it is strictly light-based and does not require the addition of any exogenous chemical agent. In addition, other light-based strategies using nonvisible light such as UVC (200 nm-280 nm wavelength) have been employed (23). A study by Dai et al. investigated the effectiveness of UVC on mouse abrasion wounds containing $S$. aureus or Pseudomonas aeruginosa (23). They found that low radiant exposures of UVC $\left(<3 \mathrm{~J} / \mathrm{cm}^{2}\right)$ effectively reduced the bacterial burden of both species (23). Although UVC is an effective approach to eliminate infection, an important consideration is

potential side effects on the surrounding mammalian tissue. Because UVC can be absorbed by host DNA, resulting in DNA damage (24), the application of longer wavelengths such as those within the blue light region have been shown to be considerably safer than UVC (24).

We next investigated whether STX photolysis by $460-\mathrm{nm}$ light was predominantly responsible for potentiating the effects of 405-nm light against $S$. aureus. We found that in a parental WT strain of $S$. aureus (Pig1), preexposure to $460-\mathrm{nm}$ light significantly improved the antimicrobial effects elicited by 405 -nm light. However, in an isogenic $\Delta$ crtM mutant of the same strain (Pig1) that does not produce STX, similar killing efficacies were observed when exposed to 405-nm light irrespective of 460-nm light preexposure. These findings strongly suggest that the improved anti-MRSA efficacy of the dual-wavelength irradiation over 405-nm aBL alone is predominantly due to STX photolysis by 460 -nm light, rendering $S$. aureus more susceptible to 405 -nm light killing.

Given that bacterial biofilms are important factors contributing to antimicrobial resistance, as well as the recalcitrant nature of MRSA infections $(25,26)$, we investigated whether dual-wavelength irradiation combining $460-\mathrm{nm}$ and 405 -nm light could be a potential modality for eliminating MRSA biofilms. With 


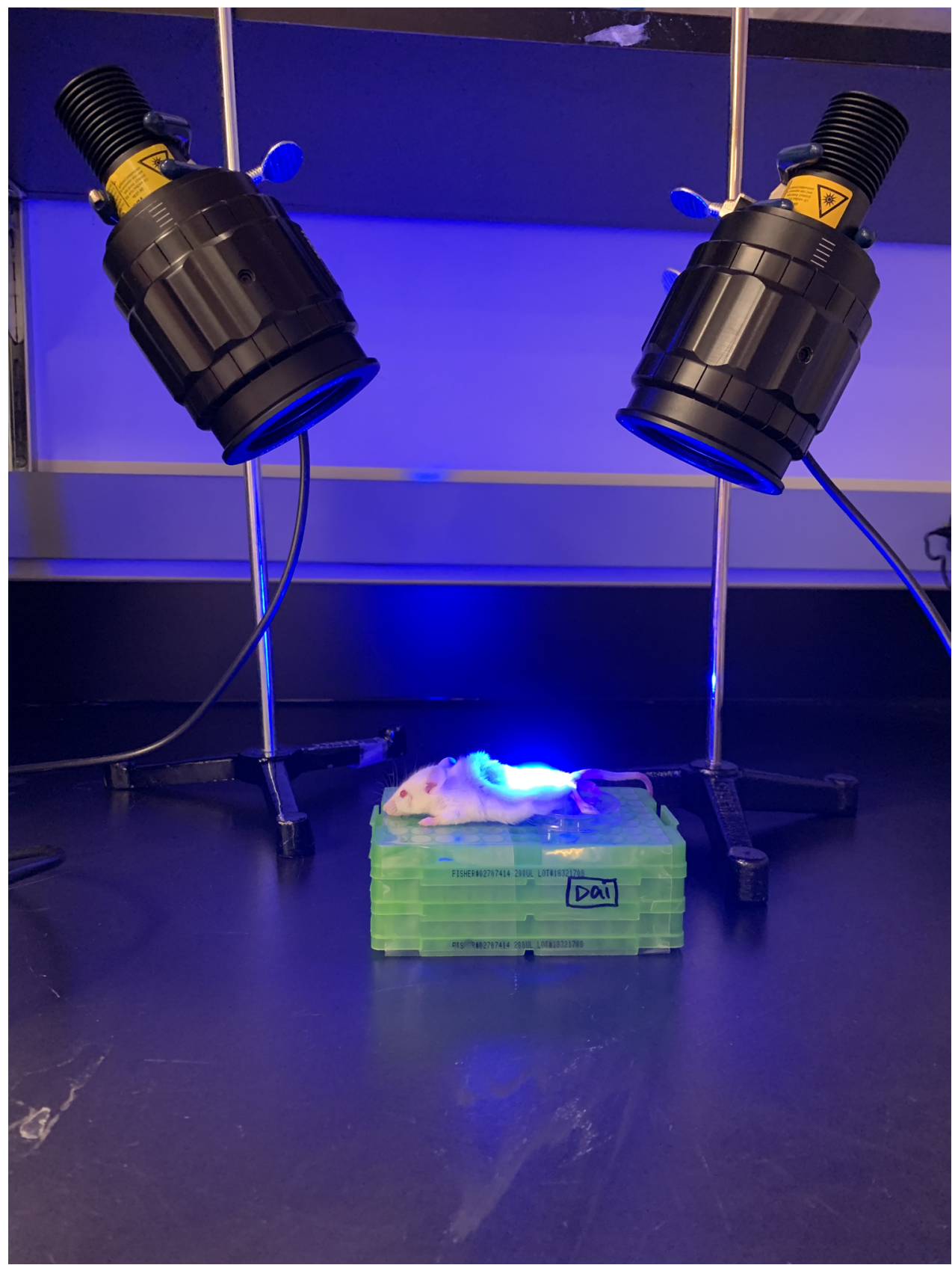

Figure 8. Experimental set-up for the treatment of MRSA within mouse wounds.

the exposure of $405-\mathrm{nm}$ light above $108 \mathrm{~J} / \mathrm{cm}^{2}$, we found that dual-wavelength irradiation significantly improved the antimicrobial effects relative to either light wavelength alone. However, no antimicrobial effects were elicited at less than or equal to $108 \mathrm{~J} / \mathrm{cm}^{2}$ of $405-\mathrm{nm}$ light in either the $405-\mathrm{nm}$ alone group or 405-nm $+460-\mathrm{nm}$ light combination-treated group. A possible explanation could be the presence of the extracellular polymeric substance (EPS) produced during biofilm formation, which may have attenuated light delivery, resulting in higher radiant exposures being required to elicit antimicrobial effects. Alternatively, MRSA cells within the EPS may be less metabolically active, reflecting a persister cell state that renders them more tolerant to antibiotic stress (27), which may explain the higher light dose required to elicit antimicrobial effects; further work is required to substantiate this hypothesis. A study by Becker demonstrated that light at $453 \mathrm{~nm}$ can increase the metabolic activity of keratinocytes in vitro, although at very low radiant exposure (28). Therefore, it is possible that $460-\mathrm{nm}$ light may have increased the metabolic activity of persister cells with biofilms, rendering them more susceptible to 405-nm light killing (at $216 \mathrm{~J} /$ 
$\mathrm{cm}^{2}$ and $324 \mathrm{~J} / \mathrm{cm}^{2}$ ). The Becker study, however, observed a biphasic dose response in which higher radiant exposures of blue light resulted in a decrease in metabolic activity (28). This is not surprising because photo-biomodulation effects are typically found at lower doses than those that would have therapeutic effects (29). Therefore, further work evaluating the dosimetry of 460-nm light on MRSA biofilms resulting in increased metabolic activity is warranted to determine its potential role in persister cell activation.

To predict the clinical therapeutic potential of the dual-wavelength irradiation approach combining 460 $\mathrm{nm}$ and 405-nm light, we investigated its efficacy in a proof-of-principle in vivo mouse skin abrasion model of infection. We established that $342 \mathrm{~J} / \mathrm{cm}^{2}$ of $460-\mathrm{nm}$ light in combination with $360 \mathrm{~J} / \mathrm{cm}^{2}$ of $405-\mathrm{nm}$ light was enough to inactivate almost $99 \%$ (1.97 $\log _{10}$ CFU reduction) of bacteria within the abrasion wounds of mice. Either wavelength of light on its own, at equivalent doses, was insufficient to result in any significant killing of MRSA compared with the untreated control. This study, therefore, demonstrated a proof-of-principle approach illustrating that the combination of 460-nm and 405-nm light may effectively reduce the MRSA burden within mouse wounds. An important limitation of this study is that using the abrasion infection as a model, coupled with the short incubation of the infection, may not necessarily be reflected in a more established or complicated soft tissue infection. Given the low penetrance of 460-nm and 405-nm light through the skin (5) $(0.5 \mathrm{~mm})$, we would expect that in the absence of more sophisticated light delivery methods, such as with the use of optical clearing agents (30) or a microneedle array for interstitial delivery (31), the efficacy of combining 460-nm + 405-nm light may be limited.

We additionally found no evidence of apoptosis (immediately after treatment, 24 hours later, or 48 hours later) resulting from combining 460-nm $+405-\mathrm{nm}$ light against naive mouse skin at a dose required to inactivate approximately $99 \%$ of MRSA within a mouse skin abrasion wound, which suggests the therapy may be safely administered. It is important to appreciate, however, that the doses required to significantly inactivate MRSA within more established wound infections may necessitate higher therapeutic doses. In addition, the results only present a qualitative assessment of safety denoted by the presence (or absence) of apoptotic cells, thus suggesting that further studies are warranted to quantitatively evaluate the safety of using 460-nm + 405-nm light against the skin. In conclusion, 460-nm + 405-nm light combination therapy may offer an effective and safe approach for the treatment of MRSA wound infections.

\section{Methods}

Blue light sources. Irradiations of 460-nm and 405-nm light were delivered using 2 light-emitting diodes (LEDs; M405L2 and M470L1; Thorlabs) with peak emissions of $460 \mathrm{~nm}$ and $405 \mathrm{~nm}$, respectively, and a full width at half-maximum of $25 \mathrm{~nm}$. The irradiance was regulated by altering the distance of the light source aperture and the target with the use of a PM100D power/energy meter (Thorlabs).

Bacterial strains and growth conditions. The bacterial strains used in this study were clinical strains of an MRSA AF0003 strain that was isolated from an infected soldier deployed in Afghanistan and an IQ00064 strain that was isolated from an infected soldier deployed in Iraq, in addition to a bioluminescent MRSA USA300 strain (23) and an MRSA Pig1 strain (7). The isogenic $\Delta c r t M$ mutant from the $S$. aureus Pig1 strain (7) was used for mechanistic studies. With the exception of the $S$. aureus Pig1 $\Delta c r t M$ strain, all are STX producers (Supplemental Figure 1). The bacteria were cultured in brain-heart infusion (BHI) medium (agar or broth) at $37^{\circ} \mathrm{C}$ or in an orbital incubator $\left(37^{\circ} \mathrm{C} ; 180 \mathrm{rpm}\right)$.

Extraction of STX from $S$. aureus strains. All strains of $S$. aureus used in the study were initially grown overnight on BHI agar from $25 \%$ glycerol freezer stocks. Subsequently, bacterial colonies were collected with the use of a $10 \mu \mathrm{L}$ loop and suspended in $10 \mathrm{~mL}$ PBS. The CFUs were then adjusted to approximately $10^{9} \mathrm{CFU} / \mathrm{mL}$ within PBS before extraction of STX. The suspended bacteria were then centrifuged at 4000 $\times g$ for 4 minutes to pellet the cells. The supernatant was then discarded, and the pellet was suspended in $2 \mathrm{~mL}$ of $100 \%$ methanol and incubated at $55^{\circ} \mathrm{C}$ for 3 hours to ensure complete extraction of the pigment. After incubation, the cells were pelleted and the supernatant containing STX was transferred to a $1 \mathrm{~mL}$ cuvette. The absorption was then measured using an Evolution 300 UV-Vis Spectrophotometer (Thermo Fisher Scientific) in accordance with the manufacturer's instructions.

Light killing $(460 \mathrm{~nm}+405 \mathrm{~nm})$ of planktonic MRSA in vitro. Bacteria were cultured to the mid-log phase and adjusted to approximately $10^{8} \mathrm{CFU} / \mathrm{mL}$ in PBS and transferred to a $35 \times 12 \mathrm{~mm}$ dish. When necessary, bacteria were pre-irradiated with 460-nm light $\left(90-360 \mathrm{~J} / \mathrm{cm}^{2} / 100 \mathrm{~mW} / \mathrm{cm}^{2}\right)$, before $405-\mathrm{nm}$ light $(108 \mathrm{~J} /$ $\mathrm{cm}^{2} / 60 \mathrm{~mW} / \mathrm{cm}^{2}$ ) exposure. During light irradiation, the bacterial suspension was stirred using a $12-\mathrm{mm}$ magnetic bar $(20 \mathrm{rpm})$ to ensure uniform exposure of cells to aBL. Aliquots $(40 \mu \mathrm{L})$ of the suspension were 
then withdrawn at varying time points after the initiation of $405-\mathrm{nm}$ light, and the CFUs were determined by serial dilution $\left(10^{-2}-10^{-7}\right.$ dilution factors) on $\mathrm{BHI}$ agar plates as described previously (32). Experiments were performed in triplicate ( 3 independent replicates spanning 3 days).

Measuring intracellular ROS after 460-nm + 405-nm light exposure. Bacteria were cultured to the mid-log phase and adjusted to approximately $10^{9} \mathrm{CFU} / \mathrm{mL}$ in PBS, and $200 \mu \mathrm{L}$ were transferred to a 96-well plate. The suspension was irradiated with $360 \mathrm{~J} / \mathrm{cm}^{2}$ of $460-\mathrm{nm}$ light, and then $10 \mu \mathrm{M}$ of CellROX Green Reagent (Thermo Fisher Scientific) was added to the bacterial suspension and incubated for 30 minutes to ensure permeabilization of the probe into cells. The bacterial suspension containing the probe was subsequently irradiated with radiant exposures of $3.6 \mathrm{~J} / \mathrm{cm}^{2}, 7.2 \mathrm{~J} / \mathrm{cm}^{2}$, or $14.4 \mathrm{~J} / \mathrm{cm}^{2}$ of 405 -nm light (low radiant exposures selected due to limited photostability of the probe) before measuring relative fluorescence emission using a SpectraMax M5 multimode multiplate reader (Molecular Devices). The excitation/emission settings were set to $485 / 520 \mathrm{~nm}$. Untreated bacteria, $405 \mathrm{~nm}$, and measurements following 460-nm treatment (reflecting the time of 405-nm exposure) were run in parallel. Experiments were performed in triplicate ( 3 independent replicates spanning 3 days).

Light killing $(460 \mathrm{~nm}+405 \mathrm{~nm})$ of MRSA biofilms. Bacterial suspensions in BHI broth were incubated in 96-well microtiter plates (200 $\mu \mathrm{L} /$ well; approximately $\left.10^{6} \mathrm{CFU} / \mathrm{mL}\right)$, as described previously, for 48 hours to induce biofilm formation $(26,27)$. Following incubation, the wells were rinsed 3 times with $200 \mu \mathrm{L}$ PBS to ensure removal of residual medium or planktonic cells, while ensuring that the adhered biofilms remained intact. Before light irradiation, aliquots of $200 \mu \mathrm{L}$ fresh PBS were added to the wells. The biofilms were pre-irradiated with $180 \mathrm{~J} / \mathrm{cm}^{2}$ of $460-\mathrm{nm}$ light at an irradiance of $100 \mathrm{~mW} / \mathrm{cm}^{2}$ immediately before $405-\mathrm{nm}$ light exposure $\left(60 \mathrm{~mW} / \mathrm{cm}^{2}\right)$ until radiant exposures of up to $324 \mathrm{~J} / \mathrm{cm}^{2}$ were delivered. The adhered cells within the biofilms were then isolated by thoroughly scraping the appropriate wells with a pipette tip and transferring the $200 \mu \mathrm{L}$ cell/biofilm suspension to a $1.5 \mathrm{~mL}$ microcentrifuge tube. A further $200 \mu \mathrm{L}$ of PBS was added to the wells, and the biofilm isolation was repeated in triplicate to ensure adequate removal of bacterial biofilms. The collected cells were subsequently sonicated using a Branson 2510 Ultrasonic Cleaner (Marshall Scientific, LLC) to ensure that cells within biofilms became adequately dislodged for subsequent plating and CFU quantification. Experiments were conducted in biological triplicate ( 3 independent replicates spanning 3 days).

Inoculation of MRSA within a mouse skin abrasion wound. Mice (Female BALB/c) aged 6-8 weeks and weighing approximately 17-19 g were procured from Charles River Laboratories. Before producing the skin abrasion wounds in mice, mice were injected intraperitoneally with the use of a ketamine/xylazine cocktail (20 mg/kg-100 mg/kg). The mice were then shaved, and the tissue was carefully abraded within a defined $1.0-\mathrm{cm} \times 1.0-\mathrm{cm}$ area using a no. 15 sterile scalpel blade. The scraped area either did not produce any blood or bled very little. Immediately after the abrasion, a $100 \mu \mathrm{L}$ bacterial suspension containing approximately $10^{7}$ CFUs of MRSA USA300 in PBS was inoculated onto the wound and applied gently and uniformly using the side of a pipette tip and left to incubate for 3 hours before treatment. To ensure that the inoculum was left intact within the mouse abrasion wound, mice were kept anesthetized until the inoculum dried within the wound. In addition, to limit physical removal of bacteria from the wound, bacteria were isolated within cages, with no more than 2 mice per cage. Experiments were performed in septuplicate ( 7 independent replicates spanning 7 days).

Light killing $(460 \mathrm{~nm}+405 \mathrm{~nm})$ of MRSA within mouse skin abrasion wounds. Three hours after inoculation of mouse abrasion wounds, MRSA was treated within mouse wounds. During light exposure in vivo, an adjustable collimator was attached to each light source, 460-nm and 405-nm light, and the LEDs were held $16 \mathrm{~cm}$ from the target, angled at an approximately $45^{\circ} \mathrm{C}$, and the collimated light beam was adjusted to cover the abrasion wound (see Figure 8). The 460-nm light was initially delivered onto the infected wound for 15 minutes $\left(200 \mathrm{~mW} / \mathrm{cm}^{2}\right)$ before irradiation with $405-\mathrm{nm}$ light $\left(60 \mathrm{~mW} / \mathrm{cm}^{2}\right)$. After the $405-\mathrm{nm}$ light was initiated, the $460-\mathrm{nm}$ light irradiance was lowered to $30 \mathrm{~mW} / \mathrm{cm}^{2}$ and exposed continuously during 405-nm light illumination (90-minute duration). The purpose of continuous exposure of 460-nm light was to ensure that continuous photolysis occurred during 405-nm illumination, as it was found that in a medium that supports MRSA growth (i.e., in vivo), STX can become replenished when 460 -nm light exposure ceases (9). The total radiant exposure of 460-nm light was $342 \mathrm{~J} / \mathrm{cm}^{2}$ and $405-\mathrm{nm}$ light was $360 \mathrm{~J} / \mathrm{cm}^{2}$ (reflecting a 105 -minute treatment time). A total of 7 mice were included for each group, and experiments were performed over 7 days (1 mouse for each group per day).

Quantification of bacterial CFUs in mouse wounds after light treatment. Immediately after treatment, mice were euthanized in accordance with standard procedures, and the $1-\mathrm{cm} \times 1-\mathrm{cm}$ infected skin tissue was isolated and homogenized in $1 \mathrm{~mL}$ PBS within a FastPrep lysing matrix tube A (MP Biomedicals) in a FastPrep-24 Classic Instrument (MP Biomedicals). Samples were homogenized for 5 minutes, with 60 sec- 
ond intervals, and samples were placed on ice for 5 minutes in between homogenization cycles to limit heat generation. After homogenization, the CFUs were determined by serial dilution $\left(10^{-2}-10^{-7}\right.$ dilution factors $)$ on BHI agar plates as described previously (32).

TUNEL assay to detect apoptotic cells in mouse skin treated with 460-nm + 405-nm light. The presence of apoptotic cells that resulted from the dual-wavelength irradiation therapy was determined in healthy mouse skin as described previously (33). In brief, skin from the mouse was isolated at 0 and 24 hours, following the treatment with 460-nm light $\left(342 \mathrm{~J} / \mathrm{cm}^{2}\right)$ and $405 \mathrm{~nm}\left(360 \mathrm{~J} / \mathrm{cm}^{2}\right)$. An untreated skin sample was also included as the control and was immediately fixed in 10\% phosphate-buffered formalin (Thermo Fisher Scientific) for 48 hours. Tissue sections ( $4 \mu \mathrm{m}$ thick) were then analyzed with the DeadEnd Fluorometric TUNEL System (Promega) in accordance with the manufacturer's instructions. Fluorescence images were visualized with the use of the NanoZoomer S60 digital slide scanner where a fluorescein isothiocyanate was used as the fluor and DAPI as the nuclear counterstain. In addition, a section treated with DNase I (RQ1 RNAsefree DNase, Promega), which induces significant DNA damage (34), served as a positive control mouse skin. Fluorescence images were visualized with the use of the NanoZoomer S60 digital slide scanner where a fluorescein isothiocyanate was used as the fluor and DAPI as the nuclear counterstain. In addition, a section treated with DNase I (RQ1 RNAsefree DNase, Promega), which induces significant DNA damage, served as a positive control.

Statistics. Data were presented as the mean \pm SEM, with differences between means being compared for significance, where appropriate, by either a paired $t$ test or 1-way ANOVA. $P$ values of less than 0.05 were considered significant.

Study approval. All animal procedures were approved by the Institutional Animal Care and Use Committees of Massachusetts General Hospital (protocol number: 2015N000187) in accordance with NIH guidelines.

\section{Author contributions}

TD proposed the concept of dual-wavelength photo-killing of MRSA. LGL and XG established a proof-of-principle for the therapeutic modality. LGL performed in vitro and in vivo studies. JXC and DCH helped provide mechanistic insights into the dual-wavelength photo-killing approach, aiding in the study design. TD and LGL designed experiments and wrote the paper.

\section{Acknowledgments}

We would like to thank Tammy Kielian at the University of Nebraska Medical Center, Omaha, Nebraska, USA, for providing the MRSA USA300 strain. We would like to thank Clinton Murray at Brooke Army Medical Center, Fort Sam Houston, Texas, USA, for providing the clinical MRSA strain isolate from a soldier in Afghanistan. In addition, we would like to thank George Y. Liu from Cedars-Sinai Medical Center for providing the $S$. aureus Pig1 strain and its isogenic $\Delta c r t M$ mutant. The study was supported in part by the NIH (R01AI123312 to TD) and the US Department of Defense (FA9550-17-1-0277 to TD). LL was supported by an American Society for Laser Medicine and Surgery Research Grant (grant no. BS.F04.18).

Address correspondence to: Tianhong Dai, Wellman Center for Photomedicine, Massachusetts General Hospital, 55 Fruit Street, Boston, Massachusetts 02114, USA. Phone: 617.726.6169; Email: tdai@mgh.harvard.edu.

1. Jackson N, Czaplewski L, Piddock LJV. Discovery and development of new antibacterial drugs: learning from experience? $J$ Antimicrob Chemother. 2018;73(6):1452-1459.

2. Kmietowicz Z. Few novel antibiotics in the pipeline, WHO warns. BMJ. 2017;358:j4339.

3. Hardgrib N, Wang M, Jurik AG, Petersen KK. Life-threatening MRSA sepsis with bilateral pneumonia, osteomyelitis, and septic arthritis of the knee in a previously healthy 13-year-old boy: a case report. Acta Radiol Open. 2016;5(10):2058460116677180.

4. [No authors listed]. World Health Organization. Antimicrobial Resistance. https://www.who.int/health-topics/antimicrobial-resistance. Accessed May 6, 2020.

5. Wang Y, et al. Antimicrobial blue light inactivation of pathogenic microbes: State of the art. Drug Resist Updat. 2017;33-35:1-22.

6. Kumar A, Ghate V, Kim MJ, Zhou W, Khoo GH, Yuk HG. Antibacterial efficacy of 405, 460 and 520 nm light emitting diodes on Lactobacillus plantarum, Staphylococcus aureus and Vibrio parahaemolyticus. J Appl Microbiol. 2016;120(1):49-56.

7. Liu GY, et al. Staphylococcus aureus golden pigment impairs neutrophil killing and promotes virulence through its antioxidant activity. J Exp Med. 2005;202(2):209-215.

8. Leejae S, Hasap L, Voravuthikunchai SP. Inhibition of staphyloxanthin biosynthesis in Staphylococcus aureus by rhodomyrtone, a novel antibiotic candidate. J Med Microbiol. 2013;62(pt 3):421-428.

9. Dong PT, et al. Photolysis of Staphyloxanthin in methicillin-resistant Staphylococcus aureus potentiates killing by reactive oxygen species. Adv Sci (Weinh). 2019;6(11):1900030.

10. Barneck MD, et al. Violet 405-nm light: a novel therapeutic agent against common pathogenic bacteria. J Surg Res. 2016;206(2):316-324. 
11. Zhang J, Suo Y, Zhang D, Jin F, Zhao H, Shi C. Genetic and virulent difference between pigmented and non-pigmented Staphylococcus aureus. Front Microbiol. 2018;9:598.

12. Terao J, Minami Y, Bando N. Singlet molecular oxygen-quenching activity of carotenoids: relevance to protection of the skin from photoaging. J Clin Biochem Nutr. 2011;48(1):57-62.

13. Enwemeka CS, Williams D, Enwemeka SK, Hollosi S, Yens D. Blue 470-nm light kills methicillin-resistant Staphylococcus aureus (MRSA) in vitro. Photomed Laser Surg. 2009;27(2):221-226.

14. Makdoumi K, Hedin M, Bäckman A. Different photodynamic effects of blue light with and without riboflavin on methicillin-resistant Staphylococcus aureus (MRSA) and human keratinocytes in vitro. Lasers Med Sci. 2019;34(9):1799-1805.

15. Makdoumi K, Bäckman A, Mortensen J, Crafoord S. Evaluation of antibacterial efficacy of photo-activated riboflavin using ultraviolet light (UVA). Graefes Arch Clin Exp Ophthalmol. 2010;248(2):207-212.

16. Makdoumi K, Bäckman A. Photodynamic UVA-riboflavin bacterial elimination in antibiotic-resistant bacteria. Clin Experiment Ophthalmol. 2016;44(7):582-586.

17. Josewin SW, Ghate V, Kim MJ, Yuk HG. Antibacterial effect of $460 \mathrm{~nm}$ light-emitting diode in combination with riboflavin against Listeria monocytogenes on smoked salmon. Food Control. 2018;84:354-361.

18. Zanetti-Polzi L, Aschi M, Daidone I, Amadei A. Theoretical modeling of the absorption spectrum of aqueous riboflavin. Chem Phys Lett. 2017;669:119-124.

19. O'kane DJ. The synthesis of riboflavin by Staphylococci. J Bacteriol. 1941;41(4):441-446.

20. Chaffin DO, Taylor D, Skerrett SJ, Rubens CE. Changes in the Staphylococcus aureus transcriptome during early adaptation to the lung. PLoS One. 2012;7(8):e41329.

21. Briggs T, et al. Antimicrobial photodynamic therapy-a promising treatment for prosthetic joint infections. Lasers Med Sci. 2018;33(3):523-532.

22. Vecchio D, et al. Bacterial photodynamic inactivation mediated by methylene blue and red light is enhanced by synergistic effect of potassium iodide. Antimicrob Agents Chemother. 2015;59(9):5203-5212.

23. Dai T, Garcia B, Murray CK, Vrahas MS, Hamblin MR. UVC light prophylaxis for cutaneous wound infections in mice. Antimicrob Agents Chemother. 2012;56(7):3841-3848.

24. Kleinpenning MM, Smits T, Frunt MH, van Erp PE, van de Kerkhof PC, Gerritsen RM. Clinical and histological effects of blue light on normal skin. Photodermatol Photoimmunol Photomed. 2010;26(1):16-21.

25. Hall CW, Mah TF. Molecular mechanisms of biofilm-based antibiotic resistance and tolerance in pathogenic bacteria. FEMS Microbiol Rev. 2017;41(3):276-301.

26. Dastgheyb S, Parvizi J, Shapiro IM, Hickok NJ, Otto M. Effect of biofilms on recalcitrance of staphylococcal joint infection to antibiotic treatment. J Infect Dis. 2015;211(4):641-650.

27. Koo H, Allan RN, Howlin RP, Stoodley P, Hall-Stoodley L. Targeting microbial biofilms: current and prospective therapeutic strategies. Nat Rev Microbiol. 2017;15(12):740-755.

28. Becker, A. Influence of photobiomodulation with blue light on the metabolism, proliferation and gene expression of human keratinocytes. Heidelberg University. https://doi.org/10.11588/heidok.00023702. Published November 14, 2017. Accessed May 6, 2020.

29. Zein R, Selting W, Hamblin MR. Review of light parameters and photobiomodulation efficacy: dive into complexity. J Biomed Opt. 2018;23(12):1-17.

30. Guo X, et al. In vivo quantification of propylene glycol, glucose and glycerol diffusion in human skin with optical coherence tomography. Laser Phys. 2010;20:1849-1855.

31. Kim M, et al. Optical lens-microneedle array for percutaneous light delivery. Biomed Opt Express. 2016;7(10):4220-4227.

32. Jett BD, Hatter KL, Huycke MM, Gilmore MS. Simplified agar plate method for quantifying viable bacteria. BioTechniques. 1997;23(4):648-650.

33. Wang Y, et al. Antimicrobial blue light inactivation of gram-negative pathogens in biofilms: in vitro and in vivo studies. $J$ Infect Dis. 2016;213(9):1380-1387.

34. Majtnerová P, Roušar T. An overview of apoptosis assays detecting DNA fragmentation. Mol Biol Rep. 2018;45(5):1469-1478 